\title{
On the Origin of Excess Thermodynamic Quantities in Liquid Mixtures
}

\author{
F. Aliotta, R.C. Ponterio and F. Saija \\ Istituto per i Processi Chimico-Fisici del CNR, Sede di Messina, Contrada Papardo, Salita Sperone, 98158 Messina - Italy \\ e-mail: aliotta@me.cnr.it - ponterio@me.cnr.it - saija@me.cnr.it
}

Résumé - Sur l'origine des quantités thermodynamiques en excès dans les mélanges de liquides - La nature des interactions entre les composants de mélanges liquides a été étudiée par diffusion Brillouin de la lumière. L'étude a porté sur plusieurs systèmes binaires capables de se mélanger sur toute la plage des concentrations. Les déviations de la compressibilité adiabatique par rapport au comportement idéal ont été observées dans chaque cas. Les résultats ont été interprétés dans le cadre d'un modèle théorique bien établi, à savoir le mélange par sphère dure non additive. En dépit du fait que des observations similaires ont été expliquées en termes d'une amélioration des interactions entre les molécules, l'analyse des fonctions thermodynamiques en excès indique que celles-ci sont dues principalement aux effets des volumes exclus et que les différences dans l'interaction intermoléculaire locale agissent en tant que contributions d'ordre plus élevé : cela peut être envisagé de manière générale pour les mélanges de liquides. La conclusion porte sur la réévaluation des données thermodynamiques en excès et sur leur capacité à fournir des informations directes sur les interactions intermoléculaires.

Abstract - On the Origin of Excess Thermodynamic Quantities in Liquid Mixtures - The nature of the interactions between components in liquid mixtures has been investigated by means of Brillouin light scattering. Several binary systems, able of mixing over the whole concentration range, have been studied. In each case, deviations of the adiabatic compressibility from ideal behaviour have been observed. This result has been interpreted within the framework of a well-established theoretical model, namely the nonadditive hard-sphere mixture. Despite similar findings were rationalized in terms of enhanced interactions between molecules, the analysis of excess thermodynamic functions indicates that they are mainly due to excluded volume effects and that the differences in the local intermolecular interaction act as higher order contributions: this can be a general consideration for liquid mixtures. A discussion is proposed on the revaluation of excess thermodynamic data and on their ability in providing direct information on intermolecular interactions. 


\section{INTRODUCTION}

The investigation of the excess thermodynamic properties in liquid mixtures is a commonly adopted approach to infer information about the nature of the inter-specie interaction [1-11]. In particular, the concentration dependence of the compressibility of a binary mixture is a quantity whose deviations from ideality are assumed able to give direct indications about the attractive or repulsive nature of the inter-specie potential.

It is quite obvious that the calculation of an excess quantity implies the definition of a reference ideal behaviour. For binary mixtures, it is usually accepted that ideality implies volume additivity. So the ideal volume of a binary mixture is usually written as:

$$
V_{i d}=\frac{x_{a} V_{m a}+x_{b} V_{m b}}{x_{a}+x_{b}}
$$

where $x_{a}$ and $x_{b}$ are the mole numbers of $a$ and $b$ component respectively and $V_{m a}$ and $V_{m b}$ are the corresponding molar volumes. After deriving Equation 1 respect to the pressure, the ideal concentration dependence of the adiabatic compressibility is soon obtained:

$$
\beta_{S, i d}=-\frac{1}{V}\left(\frac{\partial V}{\partial p}\right)_{S}=\beta_{a}+\left(\beta_{b}-\beta_{a}\right) \phi
$$

Equation 2 , where $\beta_{\alpha}$ and $\beta_{\beta}$ are the values of adiabatic compressibility of pure components, leads to assume that under ideal condition (no mixing volume) the (adiabatic) compressibility behaves linearly with the volume fraction $\phi(\phi$ is the volume fraction of the specie $b)$. Under such an assumption, a negative excess compressibility can be related to the establishment of a more rigid local structure (eventually associated with a lower correlation distance between unlike molecules, induced by an attractive inter-specie potential and resulting in a negative excess volume). On the contrary, a positive excess compressibility can be associated with some weakening of the original local connectivity due to a repulsive character of the intermolecular potential (eventually producing a positive mixing volume).

In spite of the apparent plausibility of Equation 2, we have to realize that it becomes a too rough approximation when liquid mixtures are taken into consideration. Equation 2, in fact, is a correct approximation only for low density gaseous mixtures [12] where molecules can be described as point-like objects and excluded volume effects are realistically negligible. It is easy to realize that, in a dense mixture, mixing contributions are to be taken into account whose relevance cannot be negligible. As an example, the mixing contribution to the Gibbs free energy of a liquid mixture will contain both entropic and enthalpic contributions, being this latter definitely non-zero also when Equation 1 is rigorously satisfied.
As a consequence, even if in a liquid mixtures some quantities can follow an ideal or almost ideal behaviour, it is quite impossible that this condition is verified by each physical parameter [13]. In particular, since the potential experienced by a molecule surrounded by like molecules will change if one of the neighbouring molecules is substituted with an unlike one, it becomes quite obvious that there is no theoretical limiting condition under which Equation 2 can be satisfied. This assertion corresponds to the trivial consideration that no zero-order model for the liquid state exists. For this reason, any attempt of adopting Equation 2 as a reference behaviour will be misleading: any liquid mixture will exhibit a deviation from Equation 2 and (at least part of) the apparent excess quantity is simply informing that the mixture is not a low-density gas.

This assertion can be directly supported by observing the concentration dependence of the compressibility as obtained from the most primitive model for binary liquid mixture, which adopts a hard-sphere potential both from like and unlike interaction [14]. In this model, the molecules of the two components are described as additive hard spheres of diameter $d_{a}$ and $d_{b}$, respectively. In such a way, the distances of closer approach are $d_{a}$ and $d_{b}$ for collisions between like molecules and $\left(d_{a}+d_{b}\right) / 2$ for collisions between unlike molecules. The total number density of the system is written as $\rho=\rho_{a}+\rho_{b}\left(\rho_{a}\right.$ and $\rho_{b}$ being the number densities of the species $a$ and $b$, respectively) and depends on the packing fraction $\eta$ through the relation:

$$
\rho=\frac{6}{\pi\left[(1-x) \cdot d_{a}^{3}+x \cdot d_{b}^{3}\right]} \cdot \eta
$$

being $x$ the mole fraction of the specie $b$. The virial equation of state (whose second and third coefficient are known analytically while the first one is identically equal to 1) is immediately written as [14]:

$$
\frac{p}{k_{B} T}=\sum_{i=1}^{\infty} B_{i} \rho^{i}
$$

where $p$ is the pressure, $T$ is the absolute temperature, $k_{B}$ is the Boltzmann constant.

Differentiating Equation 4 respect to the number density, the isothermal susceptibility is obtained as:

$$
\chi_{T}^{-1}=\frac{1}{k_{B} T}\left(\frac{\partial p}{\partial \rho}\right)_{T}=\sum_{i=2}^{\infty}(i-1) B_{i-1} \rho^{i-2}
$$

from which the isothermal and the adiabatic compressibilities, $\beta_{T}$ and $\beta_{S}$, can be calculated and related through the expression:

$$
\beta_{S}=\beta_{T} \frac{c_{v}}{c_{p}}=\left(\rho k_{B} T\right)^{-1} \frac{c_{v}}{c_{p}} \chi_{T}
$$


where the specific heat at constant volume for the hardsphere model is:

$$
c_{v}=\frac{3}{2} N k_{B}
$$

while the specific heat at constant pressure reads [14]:

$$
c_{p}=c_{v}+\rho\left(k_{B} T\right) \beta_{T}\left[\frac{\beta P}{\rho}\right]^{2}
$$

In Figure 1a, we report, as an example, the calculated adiabatic compressibility of a binary mixtures of hard spheres with $d_{a}=1$ and $d_{b}=2$ and for $\eta=0.2$ as a function of the molar fraction of the specie $b$. The gray area in the same figure represents the constant background due to the compressibility of the pure components that is only dependent on the value of the parameter $\eta$. The area between this background and the plot of $\beta_{S}$ represents the excess compressibility, $\beta_{e x}$, originated by the excluded volume interaction between

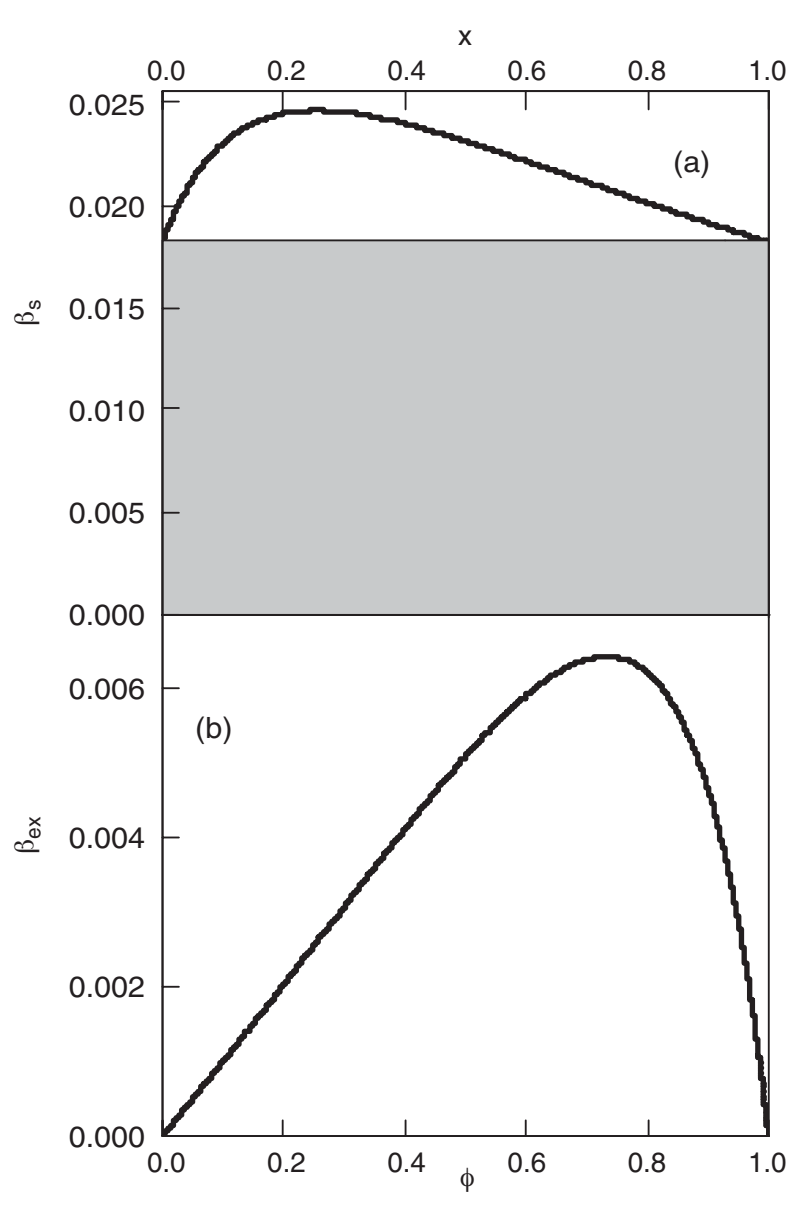

Figure 1

(a) Calculated adiabatic compressibility of binary mixtures for $d_{a}=1$ and $d_{b}=2$ and for $\eta=0.2$. Gray area: ideal mixing.

(b) Excess adiabatic compressibility for the same mixtures. unlike molecules. It is quite obvious that, under such a situation, $\beta_{e x}$ carries information about the inter-specie potential. In this case, the relevant information is the ratio $d_{b} / d_{a}$, and the value of this quantity is that one could obtain from the analysis of the concentration dependence of $\beta_{e x}$. In Figure $1 \mathrm{~b}$, we report the plot of $\beta_{e x}$ as a function of the volume fraction $\phi$ of the specie $b$. This plot corresponds to the one that can be calculated from experimental data when Equation 2 is adopted as reference. The positive excess contribution observed for the hard-sphere mixture can represent the offset behaviour which deviation from can be studied to infer information about contributions to the inter-specie potential other then excluded volume.

This choice is equivalent to assume that the regular solution (following Hildebrand $[15,16]$, it is a solution in which the two components are fully random mixed) is the most simple model to adopt for a liquid mixture, since ideal mixing has been shown to be inadequate.

Aim of this work is to exploit the effective possibility to get physical information from the comparison of the experimental results with the indication of primitive models based on hard sphere interaction.

\section{SAMPLES AND EXPERIMENTAL DATA}

The data analyzed in the present work come from previous experimental works. In particular, we will discuss some excess adiabatic compressibility data previously obtained by Brillouin scattering experiments on Carbon Tetrachloride/ Chloroform mixtures [17], on Chloroform/Ethanol and Carbon Tetrachloride/Ethanol mixtures [17] and on mixtures of Polyethylene Glycol (PEG) of mean molecular mass $600 \mathrm{~g} / \mathrm{mole}$ (PEG600) in toluene as a function of the temperature [18]. The details about the experimental apparatus [1719], the sample preparation and the data handling procedures have been published elsewhere [17, 18]. In Figures 2 and 3, we report the dependence of the mixture densities on the molar fraction, $x$ (Figs. 2a, 3a), and on the volume fraction, $\phi$ (Figs. 3a, b), of the component $b$ (see later for details and for the calculation of the quantity $\phi$ ).

\section{DISCUSSION}

The mixture chloroform/carbon tetrachloride has been selected since it closely approaches the regular solution definition. In this mixture, in fact, both density (see Fig. 2b) and refractive index behave linear with $\mathrm{CCl}_{4}$ volume fraction. The pure components have similar molar volumes $(96.5$ $\mathrm{cm}^{3} /$ mole for $\mathrm{CCl}_{4}$ and $80.0 \mathrm{~cm}^{3} / \mathrm{mole}$ for $\mathrm{CHCl}_{3}$ ) and densities $\left(1.59 \mathrm{~g} / \mathrm{cm}^{3}\right.$ for $\mathrm{CCl}_{4}$ and $1.49 \mathrm{~g} / \mathrm{cm}^{3}$ for $\left.\mathrm{CHCl}_{3}\right)$ and the two molecules are similar, differing just for the substitution of a chlorine atom with an hydrogen. In addition, we do not 


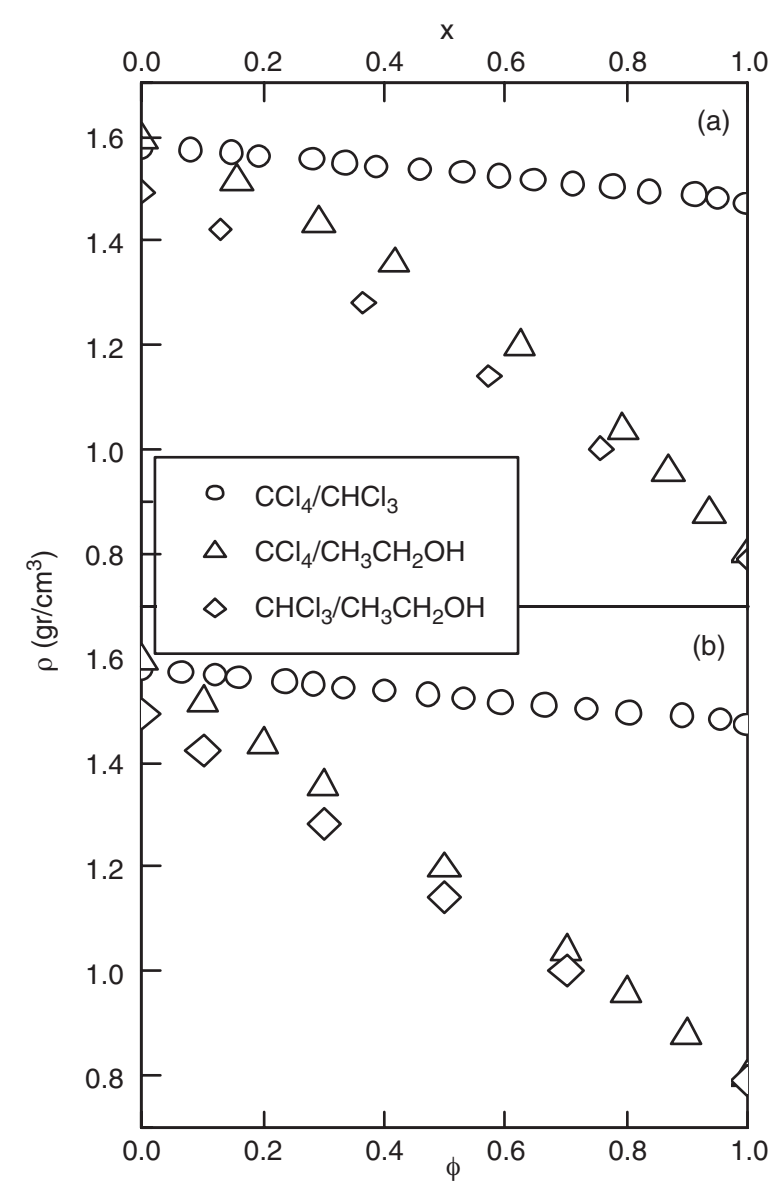

Figure 2

$x$ (a) and $\phi$-lots (b) of the experimental density at $20^{\circ} \mathrm{C}$ for some mixtures for which the volume additivity is preserved.

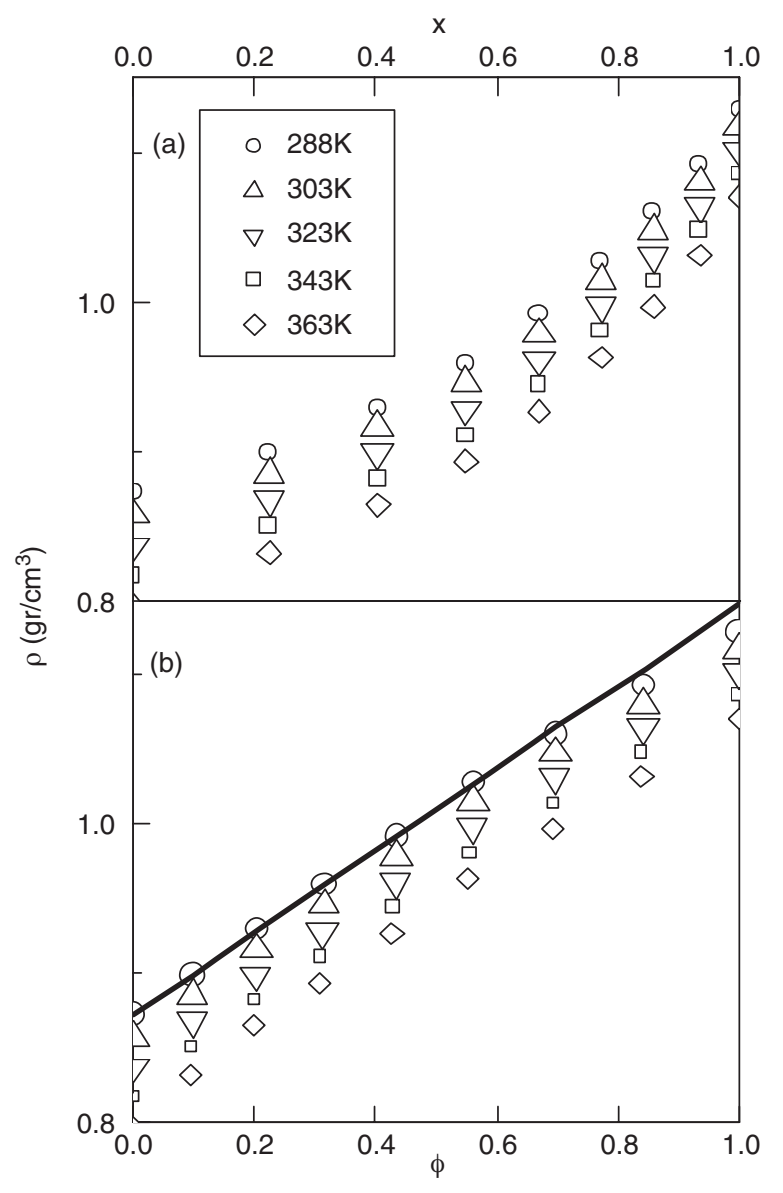

Figure 3

$x$ (a) and $\phi$-lots (b) of the experimental density for PEG600/ toluene mixtures at different temperatures. Note the deviation from the linearity (solid line) with increasing temperature. expect that the weak dipole of $\mathrm{CHCl}_{3}$ molecule is able to drive local configurations stable on the time scale of a Brillouin scattering experiment $\left(\sim 10^{-9} \mathrm{~s}\right)$.

In Figure 4a, we report the concentration dependence of the adiabatic compressibility as obtained from the experimental hypersonic velocity data. The gray area in the same figure represents, analogously to Figure 1a, the ideal behaviour, according with Equation 1. It is to be noticed, however, that on the concentration axis the volume fraction values are reported instead of the molar fraction ones as in Figure 1a. In the present case, the adoption of the $x$ scale should result in an overestimation of the excess contribution if the linear behaviour in the $x$-scale should be adopted as the reference. However, the occurrence that mixing volumes are negligible within the experimental uncertainty allows an immediate translation of the $x$-scale into the $\phi$ one. We are conscious that this argument is a trivial one, but we wish to stress this point since the assumption of linearity as a function of $x$ as reference behaviour is a practice often adopted in literature.
This choice is probably suggested by the occurrence that the values of the volume fractions cannot be ever immediately computed due to non-vanishing mixing volumes. However, this unjustified shortcoming can lead to misleading results. In Figure $4 \mathrm{~b}$, the calculated values of $\beta_{e x}$ are reported together with the results of their fitting with Equation 6. In adopting Equation 6 as a model fitting we have to take into account that the parameter $\eta$, only affects the amplitude of the theoretical curve. So we fixed the value of $\eta$ at a value of 0.2 . This value is low enough to ensure us that no phase separation occurs. So, the only free parameters in our procedure are the value of $d_{b} / d_{a}$ plus a prefactor introduced to adjust the amplitude of the theoretical curve. The best fitting reported in Figure $4 \mathrm{~b}$ has been obtained for $d_{b} / d_{a}=0.79$.

The situation becomes a little bit more complicated when one of the two component exhibits self association phenomena depending on the density. Simple examples are represented by the mixtures $\mathrm{CCl}_{4}$ /linear alcohols [17]. Linear alcohols, in fact are able to sell aggregate, via hydrogen 
bonging, in linear polymer like chains. The extent of the alcoholic chain is dependent by the kinetic equilibrium between hydrogen-bonding breaking and reforming mechanism which is dependent on both temperature and concentration. As a consequence, we can imagine that the concentration dependence of the mean aggregation size should affect the excluded volume interaction contributing to the observed excess compressibility. In Figure 5a, we report the adiabatic compressibility for $\mathrm{CCl}_{4} / \mathrm{CH}_{3} \mathrm{CH}_{2} \mathrm{OH}$ mixtures. The grey area in the same figure has the same meaning of Figure 4.

The excess adiabatic compressibility for the same mixtures is reported in Figure 5b. The dashed line in the same figure represents the best fit of the experimental data with Equation 6. $\left(\eta=0.2 ; d_{b} / d_{a}=0.69\right)$. It appears evident that, even if Equation 6 turns able to reproduce the main features of the experimental data, it slightly overestimates the excess compressibility for mixtures with high alcohol content. A possible way to improve the fitting result is to hypothesize that in this case the simple additive hard sphere interaction is not enough to model the inter-molecular interactions in our systems. However, if we assume that also in this situation the main contribution to the excess compressibility comes from inter-specie interaction, an attempt can be made for modifying the model in order to allow a non-additive hard-sphere potential [14] when unlike molecules are taken into consideration. Following this idea, the interspecies collision diameter is rewritten as $d_{a b}=1 / 2\left(d_{a}+d_{b}\right)(1+\Delta)$, being $\Delta$ a dimensionless parameter accounting for deviations of unlike excluded-volume interactions from additivity (a negative $\Delta$ value means that molecules can approach below their hardsphere radius and will result in a positive excess compressibility while a negative values has the opposite meaning

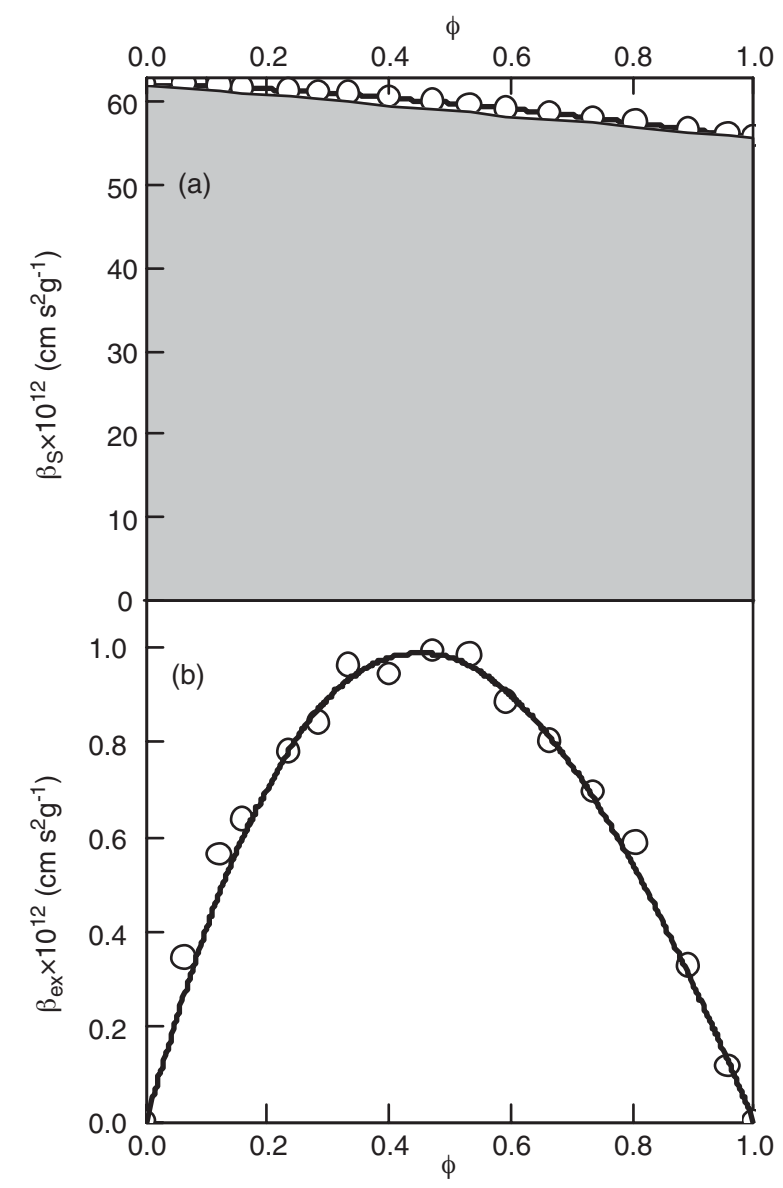

Figure 4

(a) Experimental adiabatic compressibility for $\mathrm{CCl}_{4} / \mathrm{CHCl}_{3}$ mixtures at $20^{\circ} \mathrm{C}$. (b) Excess adiabatic compressibility for the same mixtures. Continuous line: fitting with the additive hard-sphere model.

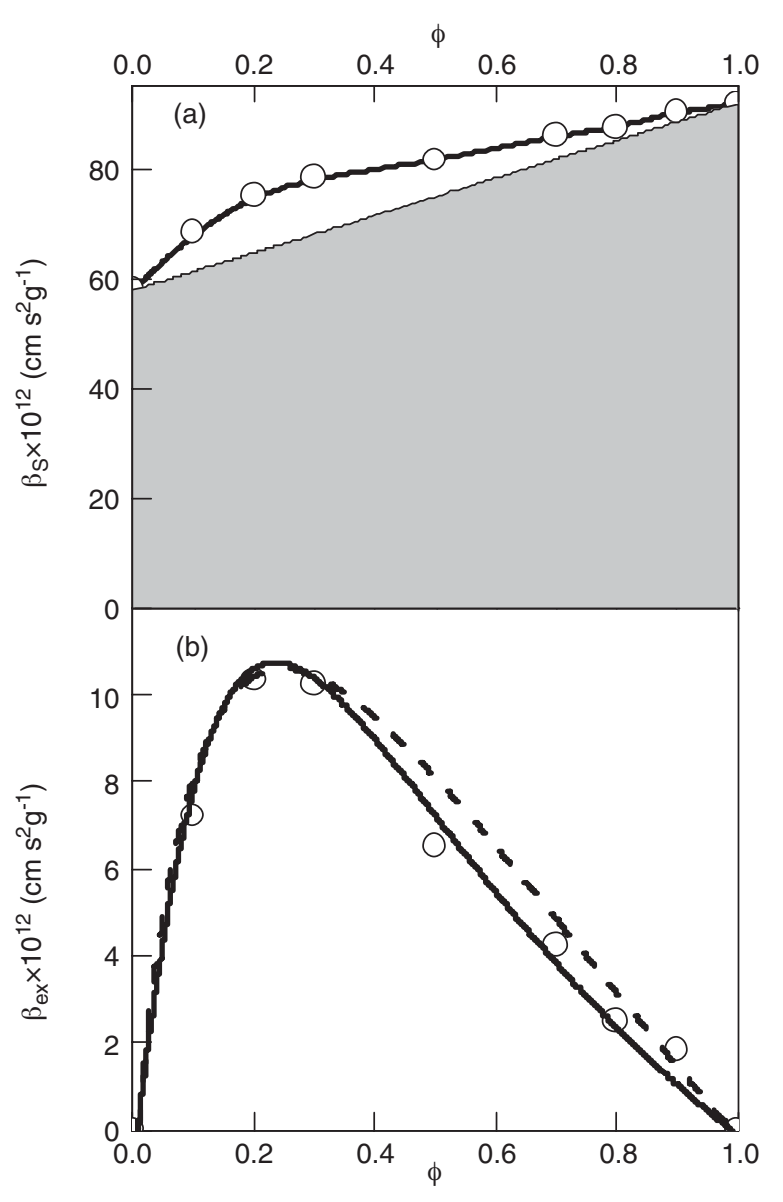

Figure 5

(a) Experimental adiabatic compressibility for $\mathrm{CCl}_{4} / \mathrm{CH}_{3} \mathrm{CH}_{2} \mathrm{OH}$ mixtures at $20^{\circ} \mathrm{C}$. (b) Excess adiabatic compressibility for the same mixtures. Dashed line: fitting with the additive hardsphere model. Continuous line: fitting with the nonadditive hardsphere model. 
producing a negative excess compressibility). Besides this point, the above model remains unaltered, being the dependence on the parameter $\Delta$ explicitly accounted through the dependence of the virial coefficients by $d_{a b}$. The continuous line in Figure $5 \mathrm{~b}$ represents the best fit of the experimental data with the non-additive hard-sphere model $\left(\eta=0.2 ; d_{b} / d_{a}\right.$ $=0.69 ; \Delta=-0.13)$. Probably, the occurrence of some electrostatic interaction between free $\mathrm{OH}$ groups and the highly polarizable $\mathrm{CCl}_{4}$ molecules, together with the existence of large hydrogen bonded aggregates, produces a less rigid local packing than that compatible with additive hard sphere interactions (note that the amplitude of the observed excess compressibility is one order of magnitude higher than that reported in Fig. 4b). However, the additional contribution to the intermolecular potential seems only able to affect the strength of the intermolecular coils without affecting the equilibrium positions of centre of mass (the density behaves linear with $\phi$, ensuring us that the additivity of volumes is preserved). In Figure 6, we report the analogous result obtained for the mixtures $\mathrm{CHCl}_{3} / \mathrm{CH}_{3} \mathrm{CH}_{2} \mathrm{OH}$. It can be observed that the values of the parameters obtained by the fitting procedure $\left(\eta=0.2 ; d_{b} / d_{a}=0.72 ; \Delta=-0.13\right)$ are very close to those we have got for $\mathrm{CCl}_{4} / \mathrm{CH}_{3} \mathrm{CH}_{2} \mathrm{OH}$. This confirms that from the point of view on which this paper is focused $\mathrm{CCl}_{4}$ and $\mathrm{CHCl}_{3}$ are very similar molecules and further support the above assumption according to which the $\mathrm{CCl}_{4} / \mathrm{CHCl}_{3}$ mixtures are a good prototype for regular solutions. In particular, it should be noticed that the values of $\beta_{S}$ for the two kinds of mixtures are very close. The main difference observed is a slightly lower $\beta_{e x}$ value for the mixtures in $\mathrm{CH}_{3} \mathrm{CH}_{2} \mathrm{OH}$ that is consistent with their overall lower density.

The situation becomes more complicated when the volumes are not additive.

However, we have to stress that for liquid mixtures the volume additivity means only that the deviations from linearity are not experimentally detectable. In fact, it is worth to remark that also a hard-sphere mixture shows an excess volume. In the previous examined cases, the occurrence that mixing volumes, are negligible within the experimental uncertainty allows an immediate translation of the $x$-scale into the $\phi$ one.

In presence of large mixing volumes only the mole fraction is an experimentally accessible quantity. Volume fraction can be only estimated according to some assumptions. A possible approach moves from the solution molar volume, $V_{M}$ (which is an experimentally determined quantity) assuming it as $[17,18]$ :

$$
V_{M}=\frac{M_{M}}{\rho}=(1-x) V_{a}^{0}+x V_{a p p}
$$

where $M_{M}=(1-\mathrm{x}) M_{a}+x M_{b}$ is the solution molar mass, $M_{a}$ and $M_{b}$ are the molar masses of the two components and $\mathrm{V}_{a}^{0}$ is the molar volume of pure component $a$ (usually the solvent). $V_{a p p}$ is the apparent molar volume of the component $b$ (the solute). This latter quantity does not correspond to any physical properties of the mixture, however it can be taken as a quantity accounting for all the contributions to the excess volume. Then, the apparent volume fraction can be calculated as:

$$
\phi=\frac{x V_{a p p}}{(1-x) V_{a}^{0}+x V_{a p p}}
$$

In Figure 7, we report the excess volumes, $V_{e x}$, calculated at different temperatures, for toluene/PEG600 mixtures [18]. $V_{e x}$ seems almost independent of the temperature and only a very moderate shift of the peak position towards a higher concentration is observed when the temperature increases. Following the approach currently adopted in literature one can be lead to conclude that a positive excess volume is

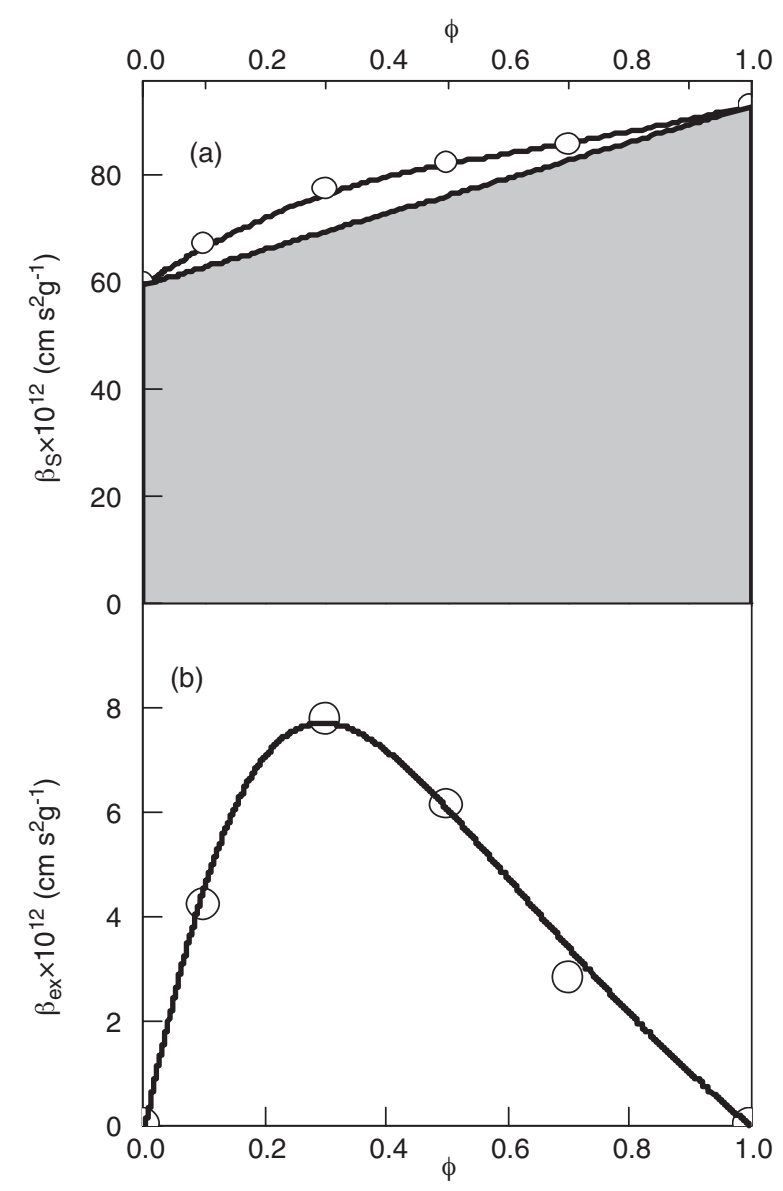

Figure 6

(a) Experimental adiabatic compressibility for $\mathrm{CHCl}_{3} / \mathrm{CH}_{3} \mathrm{CH}_{2} \mathrm{OH}$ mixtures at $20^{\circ} \mathrm{C}$. (b) Excess adiabatic compressibility for the same mixtures. Continuous line: fitting with the non-additive hard-sphere model. 


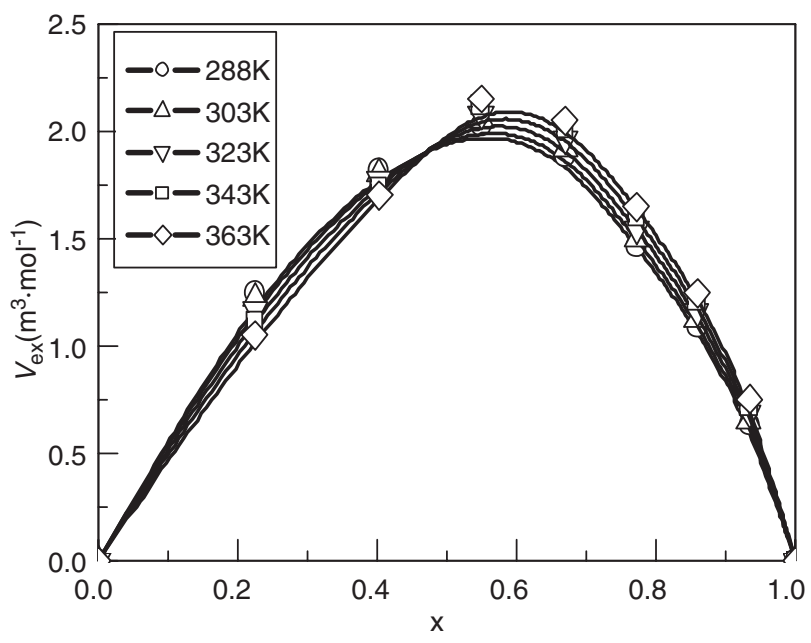

Figure 7

Calculated excess molar volumes for PEG600/toluene mixtures at different temperatures. Continuous lines are guide for eye.

the symptom of a repulsive inter-specie potential in the mixture. However, in agreement with the above considerations, this assertion only means that the overall potential is repulsive, including the intrinsic (for a dense system) contribution from excluded volume interaction. Nothing of relevant can be inferred about the nature of eventual further additional contributions. We shall come back later on this point.

After the calculation of the PEG monomer volume fraction, $\phi$, according to Equation 10, we can obtain the concentration dependence of the adiabatic compressibility. In Figure $8 \mathrm{a}$, we report the $\phi$-dependence of $\beta_{S}$ for toluene/PEG600 mixtures at different temperatures. A clear T-dependence of the curvature is observed in the $\beta_{S} v s$. $\phi$ plots that is probably related to the different expansion coefficients for the pure components. In Figure 8b, the excess compressibility is reported as a function of the volume fraction at different temperatures.

The observed change of the sign of the excess compressibility with the temperature does not seem consistent with the apparent independence of the excess molar volume by $T$. The excess data in Figure $8 \mathrm{~b}$ have been fitted with the non-additive hard sphere model. The result of the fitting are reported as continuous lines in Figure $8 \mathrm{~b}$ while in Table 1 we summarize the obtained values of the fitting parameters. The model, in spite of its obvious roughness, turns able to reproduce the main features of the observed temperature and concentration dependence of the excess compressibility, including the shaped profile observed for the lower temperature data, where the positive sign of the maximum of $\beta_{S}$ seems to suggest for the formation of a more compressible local structure. In particular, from Table 1, it becomes clear how the observed changes in the $\beta_{S}$ behaviours at different temperature

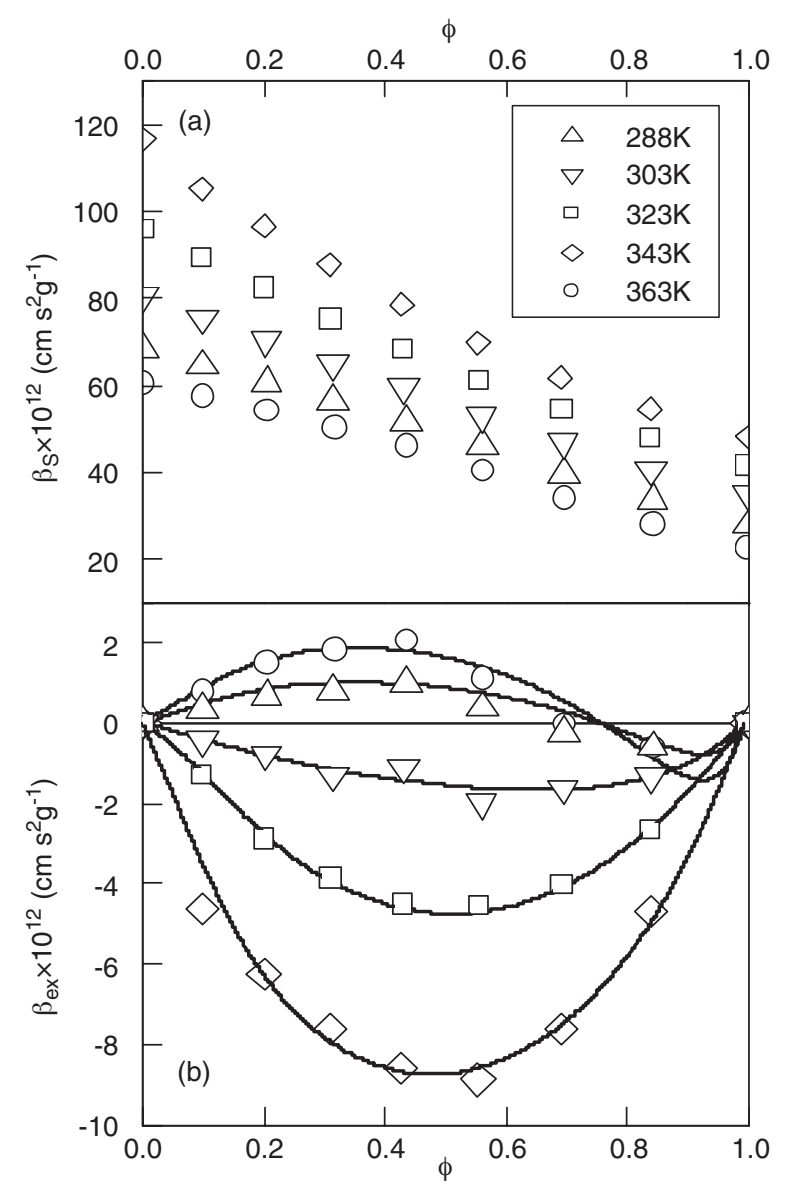

Figure 8

(a) Experimental adiabatic compressibility for PEG600/ toluene mixtures at different temperatures. (b) Excess adiabatic compressibility for the same mixtures. Continuous line: fitting with the non-additive hardsphere model.

can be reproduced assuming a moderately positive value for the $\Delta$ parameter. This means that an attractive contribution to the inter-specie potential must be taken into account besides the repulsive contribution suggested by a first inspection of Figure 7. In such a way, the excess molar volume and excess adiabatic compressibility behaviour can be interpreted consistently assuming that they are the result of a balance among different interaction potentials. In particular, the data reported in Table 1 show how a positive non-additive contribution to the inter-specie interaction diameter should be taken into account for each of the curves reported in Figure $8 \mathrm{~b}$. This contribution becomes more efficient at higher temperatures where it is able to overcome the effects of the basic additive hard-sphere contribution, depicted in Figure 1b. On one side, the interspecie interaction determines a lowering of the mixture density while, on the other side, the positive nonadditive contribution to the interaction potential makes the local structure more rigid at higher temperatures. 
TABLE 1

Parameters of the fitting of $\beta_{e x}$ for PEG600/toluene mixtures with the non-additive hardsphere model

\begin{tabular}{c|c|c|c}
\hline$T$ & $d_{b} / d_{a}$ & $\Delta$ & $\eta$ \\
\hline $288 \mathrm{~K}$ & 2.50 & 0.1270 & 0.2 \\
$303 \mathrm{~K}$ & 2.50 & 0.1270 & 0.2 \\
$323 \mathrm{~K}$ & 1.80 & 0.0710 & 0.2 \\
$343 \mathrm{~K}$ & 1.30 & 0.0139 & 0.2 \\
$363 \mathrm{~K}$ & 1.55 & 0.0560 & 0.2 \\
\hline
\end{tabular}

TABLE 2

Parameters of the fitting of $\beta_{S}$ for PEG600/toluene mixtures with the modified non-additive hard-sphere model (see text)

\begin{tabular}{c|c|c|c}
\hline$T$ & $d_{b} / d_{a}$ & $\Delta$ & $\eta$ \\
\hline $288 \mathrm{~K}$ & 2.50 & 0.085 & 0.29 \\
$303 \mathrm{~K}$ & 2.20 & 0.070 & 0.17 \\
$323 \mathrm{~K}$ & 1.50 & 0.005 & 0.12 \\
$343 \mathrm{~K}$ & 1.40 & 0.005 & 0.12 \\
$363 \mathrm{~K}$ & 1.55 & 0.022 & 0.12 \\
\hline
\end{tabular}

\section{CONCLUDING CONSIDERATIONS}

We have shown how the usual way of estimating the values of excess compressibility in binary mixtures can lead to misleading results since it does not takes into account for an intrinsic (for dense mixtures) positive excess contribution originated by the excluded volume interaction. In addition, we have shown how a primitive model based on non-additive hard-sphere interactions is able to reproduce the large variety of experimental behaviours. However, in the above reported results, we have to consider that the excess compressibilities can be affected by large errors; in fact, their values, obtained as the difference between the experimental compressibility and the ideal behaviour, estimated by Equation 2, can be large. The error can be even more large in situation as that reported in Figure 8 where the non-additivity of volumes requires some assumptions in order to calculate the approximated value of the volume fraction. As an example, in Figure 3a, it can be observed that our attempt to correct the effect of a nonnegligible mixing volume produces only approximated results: the plots of $\rho v s . \phi$ slightly deviate from linearity. For these reasons, a better choice should be the adoption of a fitting model able to reproduce the concentration behaviour of $\beta_{S}$ instead of $\beta_{e x}$ (that in turns means a model able to reproduce the compressibility of pure components). From this point of view, the hard-sphere approach is not suitable because the compressibilities of the pure components are described as independent on the specie (independent on the sphere diameter) and only dependent on the packing factor $\eta$.

However, we can try a rough attempt substituting the density described in terms of Equation 3 with a quantity proportional to the experimental density.

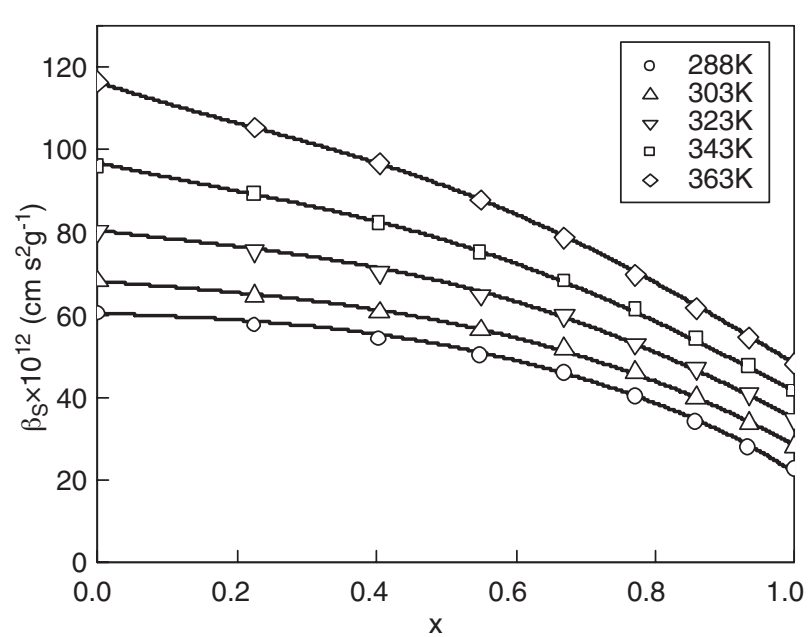

Figure 9

Experimental adiabatic compressibility for PEG600/toluene mixtures at different temperatures. Continuous lines: fitting results with the modified (see text) non-additive hardsphere model.

We have fitted the experimental curves of $\rho$ vs. $x$ (Fig. 3b) with 3rd order polynomials. The obtained values have been used in the calculus of the virial coefficients and, then, the experimental data have been fitted with Equation 6. The fitting results are reported as continuous line in Figure 9 while the obtained values of the fitting parameters are summarized in Table 2 . The value of the $\eta$ parameter is now more critical than what it was in the fitting of the excess compressibility, since it determines the difference in the compressibility of pure components. The obtained values of $\eta$ are not meaningful since they include a multiplicative factor to match the experimental amplitudes. However, the concentration dependence of $\eta$ can carry physical information about the mixture. In particular, while the values of the $d_{b} / d_{a}$ and $\Delta$ parameters are not to much different from the previously obtained ones, an increasing of the values of $\eta$ at the lower temperatures is observed. This increasing of the packing factor is consistent with usual increasing of the density with temperature lowering. In such a way, the temperature dependence of the excess compressibility reported in Figure 8 can be just the effect of the temperature dependence of the mixture density (different for each component).

We are conscious that this last approach is a very rough one and that we are forcing the non-additive hardsphere model beyond its limits. However, we feel that the data reported in Figure 9 and in Table 2 can be useful for suggesting new approaches suitable to extract realistic information from excess compressibility data. 


\section{REFERENCES}

1 Aliotta F., Magazù S., Maisano G., Majolino D., Migliardo P., Venuto S. (1993) Sound velocity and hydration phenomena in aqueous polymeric solutions, Mol. Phys. 78, 2, 421-435.

2 Paraskevopoulos G.C., Missen R.W. (1962) Thermodynamic properties of solutions of alcohols and carbon tetrachloride. Part 1. Free energies and volumes of mixing, T. Faraday Soc. $\mathbf{5 8}, 869-878$.

3 Otterstedt J.E.A., Missen R.W. (1962) Thermodynamic properties of solutions of alcohols and carbon tetrachloride. Part 2. Heats and entropies of mixing, T. Faraday Soc. 58, 879-889.

4 Arce A., Rodil E., Soto A. (2006) Physical and excess properties for binary mixtures of 1-methyl-3-octylimidazolium tetrafluoroborate, [Bmim] [BF4], ionic liquid with different alcohols, J. Sol. Chem. 35, 1, 63-78.

5 Pochylski M., Aliotta F., Blaszczak Z., Gapinski J. (2006) Structuring effects and hydration phenomena in poly(ethylene glycol)/water mixtures investigated by Brillouin scattering, J. Phys. Chem. B 110, 41, 20533-20539.

6 Zafarani-Moattar M.T., Shekaari H. (2006) Application of Prigogine-Flory-Patterson theory to excess molar volume and speed of sound of 1-n-butyl-3-methylimidazolium hexafluorophosphate or 1-n-butyl-3-methylimidazolium tetrafluoroborate in methanol and acetonitrile, J. Chem. Thermodyn. 38, $11,1377-1384$.

7 Pfeiffer H., Heremans K. (2005) The sound velocity in ideal liquid mixtures from thermal volume fluctuations, Chem. Phys. Chem. 6, 4, 697-705.

8 Ali A., Nain A.K. (2002) Ultrasonic study of molecular interaction in binary liquid mixtures at 30 degrees C, Promana J. Phys. 58, 4, 695-701.

9 Kadam U.B., Hiray A.P, Sawant A.B., Hasan M. (2006) Densities, viscosities, and ultrasonic velocity studies of binary mixtures of chloroform with propan-1-ol and butan-1ol at (303.15 and 313.15) K, J. Chem. Eng. Data 51, 1, 60-63.

10 Aliotta F., Musso M., Ponterio R., Saija F., Salvato G. (2004) Evidence of heterogeneous aggregation in methanol/CCl4 mixtures: A Brillouin scattering investigation, J. Phys. Chem. B 108, 34, 12972-12977.

11 Aliotta F., Ponterio R., Salvato G., Musso M. (2004) Brillouin scattering evidence of nonideal mixing in methanol/CCl4 mixtures, J. Phys. Chem. B 108, 2, 732-736.

12 Hirschfelder J.O., Curtiss C.F., Bird R.B. (1966) Molecular Theory of Gases and Liquids, Wiley, New York.

13 Flory P.J. (1965) Statistical Thermodynamics of Liquid Mixtures, J. Am. Chem. Soc. 87, 1833-1838.

14 Boublík T., Nezbeda I. (1986) $P-V-T$ Behaviour of Hard Body Fluids. Theory and Experiment, Collect. Czech. Chem. C. 51, 2301-2432.

15 Hildebrand J.H. (1947) The entropy of solution of molecules of different size, J. Chem. Phys. 15, 5, 225-228.

16 Hildebrand J.H. (1951) The term "Regular Solution", Nature 168, 868 .

17 Aliotta F., Gapinski J., Pochylski M., Ponterio R.C., Saija F., Salvato G. (2007) Excess compressibility in binary liquid mixtures, J. Chem. Phys. 126, 22, 224508.

18 Pochylski M., Aliotta F., Blaszczak Z., Gapinski J. (2006) Evidences of nonideal mixing in poly(ethylene glycol)/organic solvent mixtures by Brillouin scattering, J. Phys. Chem. B 110, 1, 485-493.

19 Salvato G., Ponterio R.C., Aliotta F. (2006) New automatic system for multipass Fabry-Perot alignment and stabilization, Rev. Sci. Instrum. 77, 11, 113104 .

Final manuscript received in November 2007 Published online in April 2008

Copyright (c) 2008 Institut français du pétrole

Permission to make digital or hard copies of part or all of this work for personal or classroom use is granted without fee provided that copies are not made or distributed for profit or commercial advantage and that copies bear this notice and the full citation on the first page. Copyrights for components of this work owned by others than IFP must be honored. Abstracting with credit is permitted. To copy otherwise, to republish, to post on servers, or to redistribute to lists, requires prior specific permission and/or a fee: Request permission from Documentation, Institut français du pétrole, fax. +33147527078 , or revueogst@ifp.fr. 